\title{
Computational investigation of wall shear stress patterns on calcified aortic valve leaflets
}

\author{
Huseyin Enes Salman ${ }^{1}$, Huseyin Cagatay Yalcin ${ }^{1}$ \\ ${ }^{1}$ Biomedical Research Center, Qatar University, PO Box 2713, Doha, QATAR
}

\section{BACKGROUND}

- Aortic valve diseases affect about $25 \%$ of the population over 65 years of age.

- Aortic valve separates the left ventricle from the aorta, and consists of three half-moon shaped leaflets.

- Leaflets are highly dynamic structures which open during the ventricle contraction and close during the ventricle relaxation.

- Calcification on leaflet surfaces results in poor valve functioning which deteriorates the valve hemodynamics.

- Wall shear stresses (WSS) on the leaflet surfaces are considered to be strongly related with the initiation and progression of calcification.

\section{OBJECTIVE}

To investigate the effect of altered hemodynamics on the valve leaflet calcification, and to understand the role of WSS patterns in the progression of the aortic valve diseases.

\section{METHODOLOGY}

Computational modeling using fluid-structure interaction simulations - The problem is first defined mathematically, and the solution is approximated with numerical techniques by following the steps:

- Segmentation of medical images to construct 3D model

- Discretization of the problem domain into finite elements (Meshing)

- Applying related inlet and outlet boundary conditions considering the pulsatile flow

- Performing finite element analysis (FEA)

- Post-processing the results

- The governing equations in fluid domain:

$$
\begin{gathered}
\rho_{f} \frac{\partial \mathbf{v}}{\partial t}+\rho_{f}(\mathbf{v}-\mathbf{w}) \cdot \nabla \mathbf{v}-\nabla \cdot \boldsymbol{\tau}_{f}=\mathbf{f}_{f}^{\mathrm{B}} \\
\nabla \cdot \mathbf{v}=0
\end{gathered}
$$

$\rho_{f}$ : Mass density of fluid, $\quad \mathbf{v}$ : Fluid velocity vector, $t$ : Time

$\mathbf{w}$ : Velocity of the fluid domain (i.e. moving coordinate velocity)

$\boldsymbol{\tau}_{f}$ : Fluid stress tensor, $\quad \mathbf{f}_{f}^{\mathrm{B}}$ : Body forces

- The governing equation in solid domain:

$$
\nabla \cdot \boldsymbol{\tau}_{s}=\rho_{s} \ddot{\mathbf{d}_{s}}
$$

$\boldsymbol{\tau}_{s}$ : Solid stress tensor, $\rho_{s}$ : Mass density of the leaflets

$\ddot{\mathbf{d}}_{s}$ : Local acceleration in the solid domain

- By coupling the solutions of fluid and solid domains complex dynamic motions of leaflets are simulated.

- Blood flow velocities and WSS values are determined within the entire aortic valve domain.

- Computational results are validated by comparing with B-mode echo images.

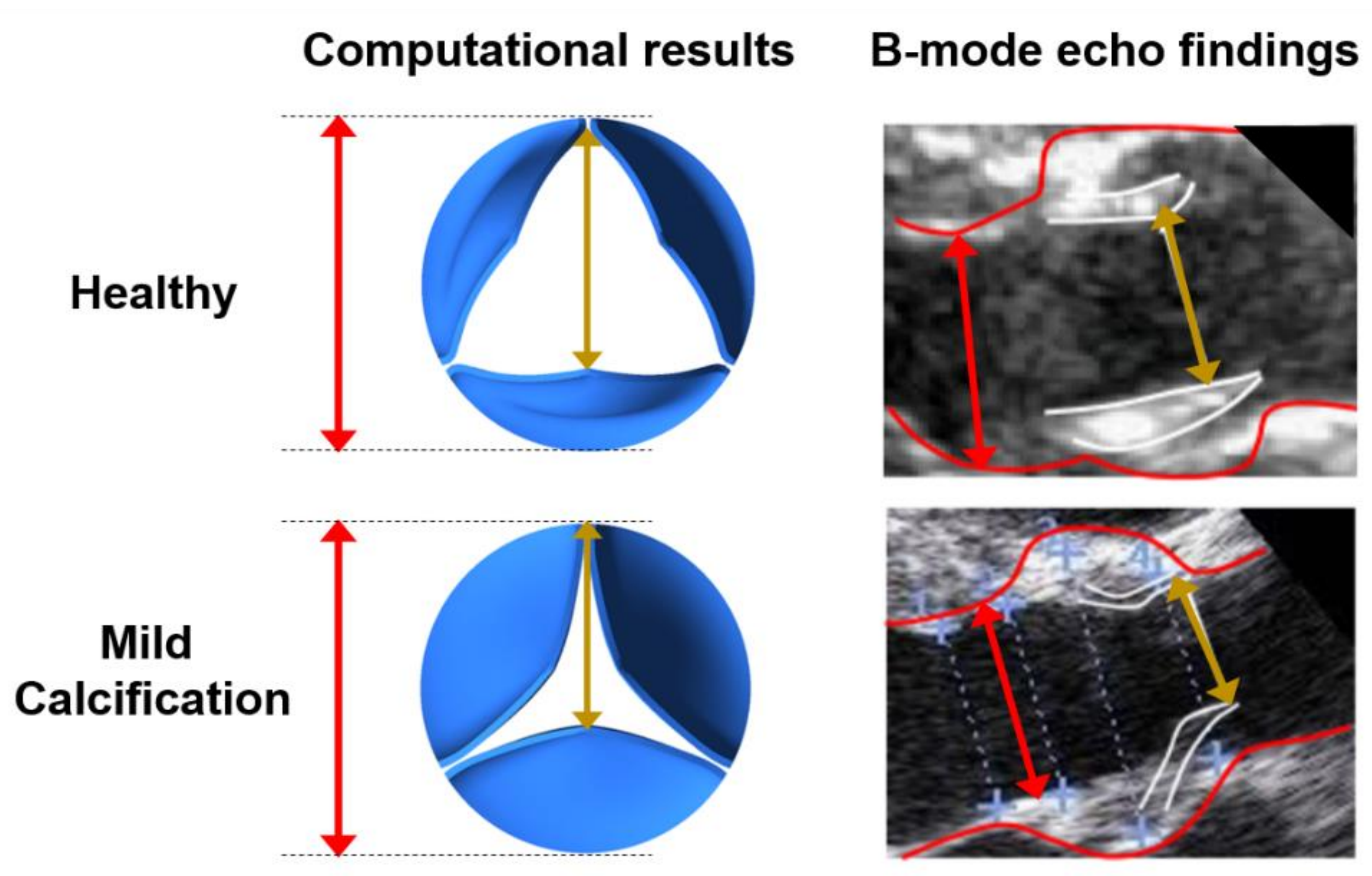

Leaflet deformation at the instant of maximum aortic valve opening

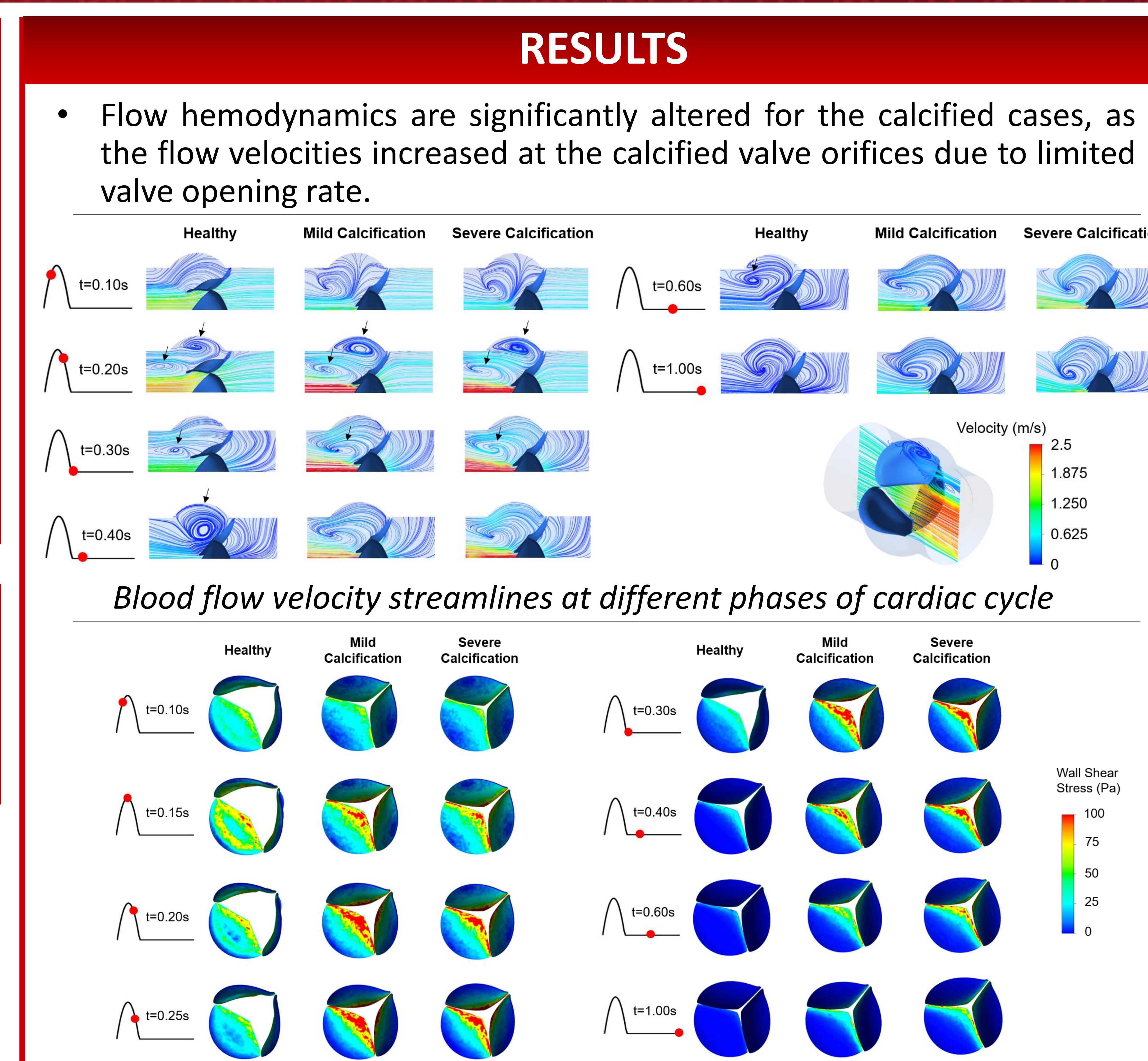

WSS patterns on ventricularis surface of the leaflet within one cardiac cycle

- In case of calcification, WSS values are increased at both surfaces of the leaflets.

- On the ventricularis surface, there is a spatially-regular WSS distribution, which gradually increase from the leaflet attachment region to the leaflet tip.

- However, a spatially-complex WSS distribution is observed on the aortic leaflet surface.

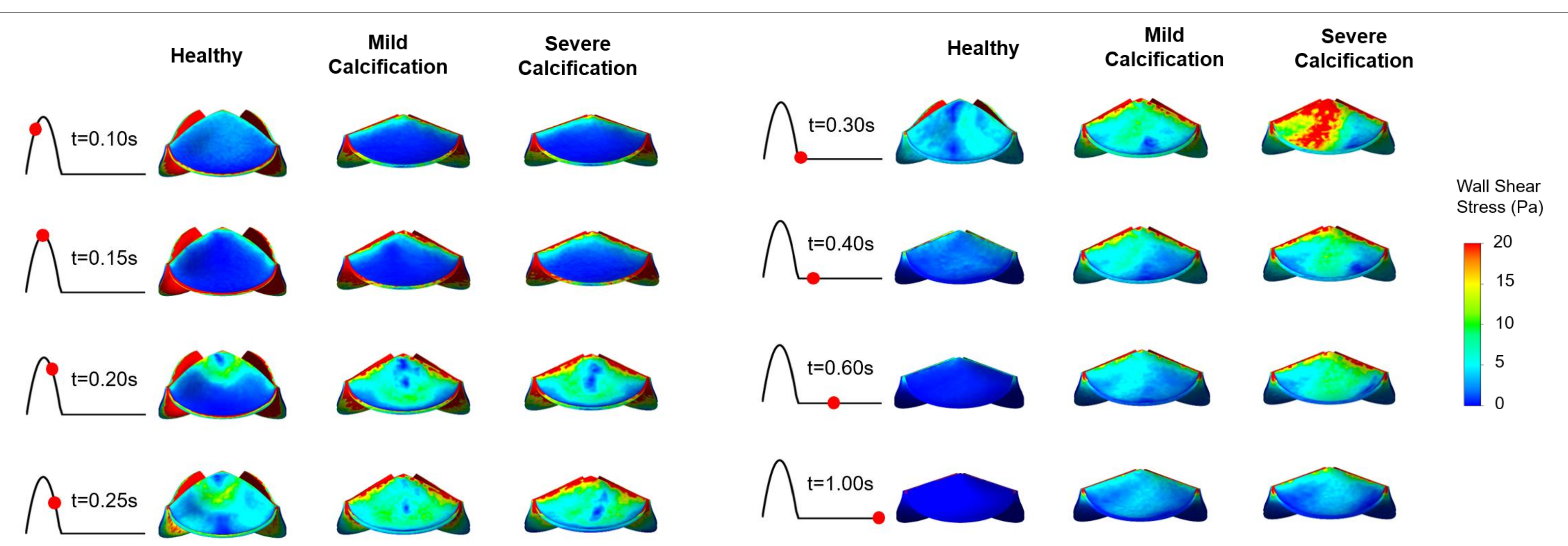

WSS patterns on fibrosa surface of the leaflet within one cardiac cycle

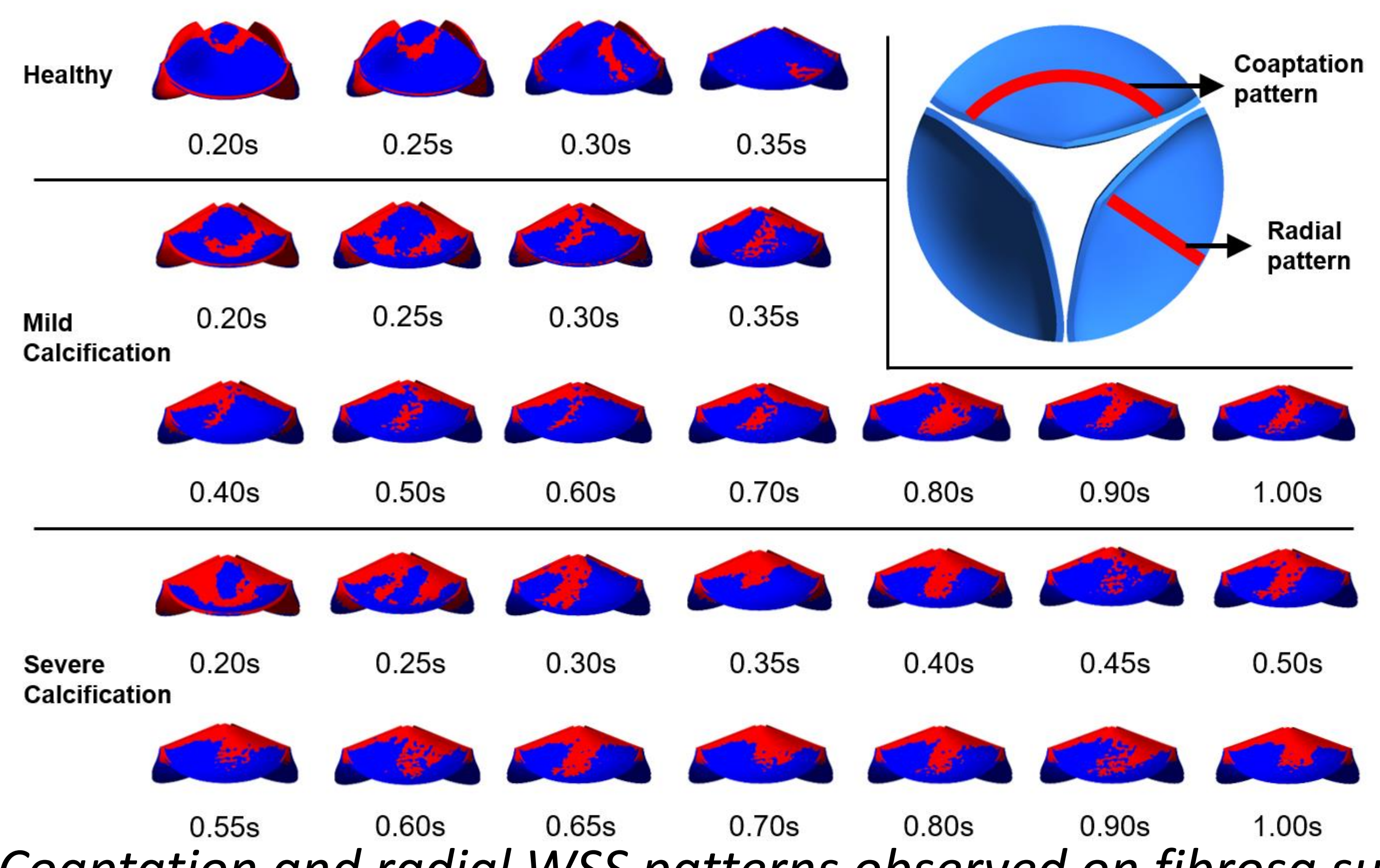

Coaptation and radial WSS patterns observed on fibrosa surface

- Radial WSS patterns become more dominant in the cardiac cycle depending on increasing leaflet stiffness due to calcification, particularly on the fibrosa surface within time range of 0.3-1.0s.

\section{CONCLUSION}

Relatively low WSS levels and spatially-complex WSS patterns on the aortic leaflet surfaces are observed as potential risk factors for the initiation and progression of aortic valve calcification. 